\title{
Solving the problem of optimal design for a two-stage reducer by using a modified evolutionary algorithm
}

\author{
Oleksandr Ustynenko ${ }^{1}$, Oleksiy Bondarenko ${ }^{1}$, and Volodymyr Serykov ${ }^{1}$ \\ ${ }^{1}$ National Technical University "Kharkiv Polytechnic Institute", Department of Theory and Computer-Aided Design of \\ Mechanisms and Machines, 61002 Kharkiv, Ukraine
}

\begin{abstract}
The work is devoted to solving the problem of selecting optimal geometric parameters of gears of a two-stage cylindrical reducer using a modified evolutionary algorithm (EA).

The statement of the problem is considered, design parameters, objective functions, limitations on design parameters are determined. This allowed us to propose a modification of EA. To generate the initial test points, it was proposed to use the LP- $\tau$ sequence, this allowed us to reduce the initial population of test points and bring EA closer to a truly "random" process.
\end{abstract}

The scheme of the proposed algorithm is considered, which gives an idea of the sequence of operations that are carried out with populations of test points at each stage of the evolutionary process.

\begin{abstract}
The solution of the specific problem of selecting optimal parameters for a serial reducer is given. The input data, numerical and functional limitations are determined, the objective functions are formed. The results of the solution are shown in several presentation formats: tabular and graphical, which allows to qualitatively interpret and analyze the results.
\end{abstract}

Conclusions are made about testing the proposed algorithm for solving a specific problem of optimal design. Further ways of improving this methodology are proposed.

\section{The actuality of the task}

General mechanical engineering uses universal and special two-stage coaxial and expanded reducers. Automotive engineering requires two- and three-shafts gearboxes [1,2], which for each (not direct) gears work as a one- and two-stage reducer.

The main problems and difficulties in the design of this type of actuators were discussed in [3].

One approach to solving problems was used pseudorandom search for $\operatorname{LP} \tau$-sequences, both alone and in combination with other methods [4,5].

Possibilities and adaptation of genetic algorithms for optimal design of gearboxes and reducers were considered in [6]. In this work several variations of genetic algorithms are given, where $\operatorname{LP} \tau$-uniformly distributed sequences were used to generate the initial populations.

The authors see the possible development of this direction and the improvement of similar approaches to solving the problems of optimal design for the specified gear drives [7-9].

Thus, solving the problem of optimal design for a two-stage reducer by using a modified evolutionary algorithm is actual applied task.

\section{Design parameters and design criteria}

Design parameters and objective functions for this task can be seen in $[3,5,10]$. But note that in the case of a non-coaxial reducers, the objective function of the criterion of minimum center distance is the sum of the center distance of the individual meshes.

\section{Limitations and functional relationships between constructive parameters}

Some limitations regarding the solution of this problem can be seen in previous work $[3,5,10]$. Here we just want to focus on the functional and numerical limitations used in the proposed algorithm to test the viability of test points.

A) For reducers, the requirement of relative equality of larger gear wheels in the meshes may be advanced. This makes it possible to ensure the proportional shape of the reducer and the evenness of the gear wheels in the oil bath:

\footnotetext{
Corresponding author: ustin1964@gmail.com
} 


$$
\max \left(d_{11}, d_{12}\right) \approx \max \left(d_{21}, d_{22}\right) .
$$

B) For the coaxial reducers, the equality of the axial distances of the individual gears must be maintained:

$$
a_{w 1}=a_{w 2} .
$$

C) The teeth number, which calculated through the generated and input parameters, must accept integers (must be natural $-N$ ), also limited to the upper and lower values for manufacturing technology reasons:

$$
z_{22} \in N ; \quad z_{\min } \leq z_{22} \leq z_{\max } .
$$

From the requirement of the overall relationship, the gear ration must not exceed a certain value $\left(u_{\max }\right)$ :

$$
u_{\mu}=\frac{\max \left(z_{\mu, 1}, z_{\mu, 2}\right)}{\min \left(z_{\mu, 1}, z_{\mu, 2}\right)} \leq u_{\max } .
$$

D) The teeth of the wheels must have the necessary contact durability and the required bending strength:

$$
\begin{aligned}
\sigma_{H \mu} & \leq \sigma_{H P \mu} ; \\
\sigma_{F \mu, k} & \leq \sigma_{F P \mu, k} .
\end{aligned}
$$

E) The condition for the absence of sharpening tooth tip pointing:

$$
s_{a \mu} \geq 0.4 \cdot m_{\mu} .
$$

F) The face width factor is also limited to extreme values:

$$
\psi_{b d \mu \min } \leq \psi_{b d \mu} \leq \psi_{b d \mu \max } .
$$

\section{Evolutionary algorithms}

Evolutionary Algorithms (EA) [6,9] are special approaches to search. They are used to solve optimization problems and modeling of states and processes through random or directional actions and processes with parameters. Such strategies are identical to the processes of selection and heredity in wildlife. EA is used as one of the mechanisms for solving multivariable problems in mechanics. EA differ from the classical methods of optimization by some features.

The main difference EA is that in the following generations, not all individuals are passed on, but only those that satisfy the conditions of existence - certain limitations put forward by the designer.

Such a population development strategy enables individuals in each next generation to obtain more and more adaptability to the requirements. The analysis of each generation goes by fairly quickly, because the number of individuals is controlled by the limitations.

Consider the EA proposed by the authors for solving the problem of rational design for two-stage reducers (Fig. 1).

To approach the natural process of evolution, it is necessary to provide a certain amount of randomnicity in the EA process. There are several opposing views in the world of science regarding the evolutionary process: one view argues that evolution is a random process, the second view argues that evolution is a directed necessity.

The authors urge not to go to extremes and consider EA as a complex process that accommodates randomnicity and necessity in certain parts. The need is determined by the numerical and functional technical and technological limitations assigned by the designer, and by randomnicity the use of a pseudorandom factor at certain stages of EA. As the latter, the authors propose to use the LP $\tau$-sequence.

It is proposed in EA to consider the following stages at the discretion of pseudorandom: generation of initial population, choice of parental pairs, crossing and mutation.

The initial population is then subjected to a viability analysis within which individuals are identified to be involved in the subsequent life cycle. When solving technical problems, this analysis corresponds to the operation of checking the compliance of the object with the technical and technological limitations, which guarantees its operability.

The selection of parental couples [6] is recommended to be performed according to the panmixia strategy.

The next step is determining how genes will be exchanged, that is, crossed and mutated. From the list of mutation operators, the authors recommend using a step mutation. Of the many possible variants of crossing, the authors chose the more convenient in terms of software implementation - multipoint crossing. The formed population must again be subject to analysis, that is, the algorithm cycle is closed.

Thus, passing a certain number of cycles, the population of viable quality individuals is gradually replenished. At the end of the cycle, we get the resulting population, which is analyzed by the value of the objective function and sorting. At the output of the algorithm we have one or more leading points that determine the solution of the problem. 


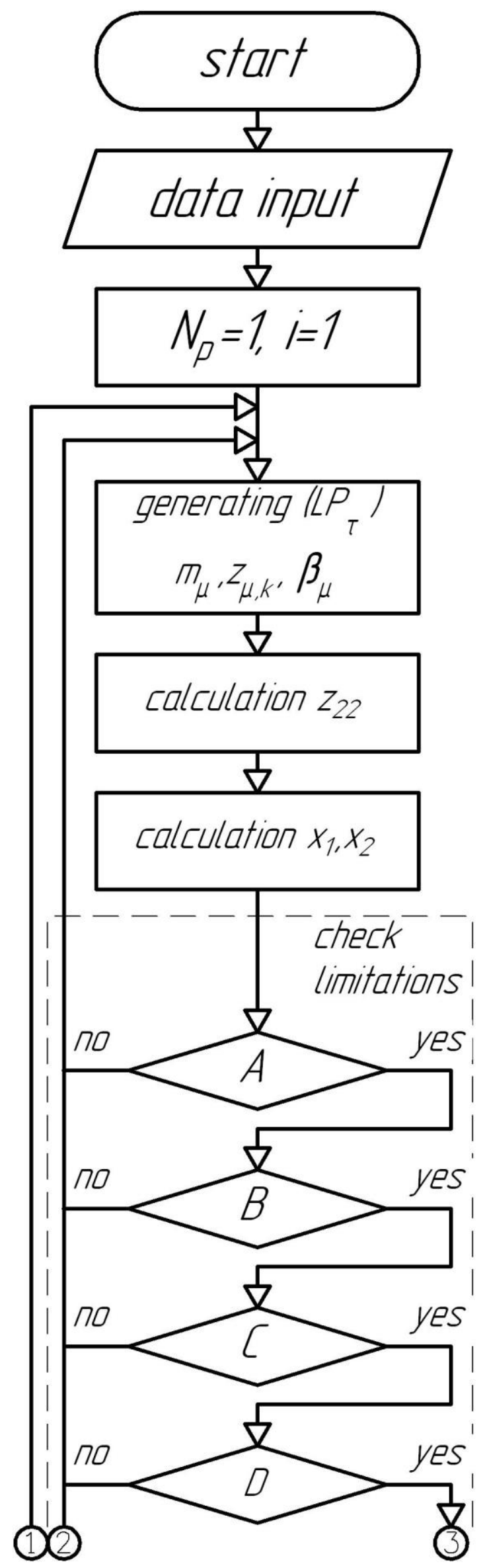

Fig. 1. EA.

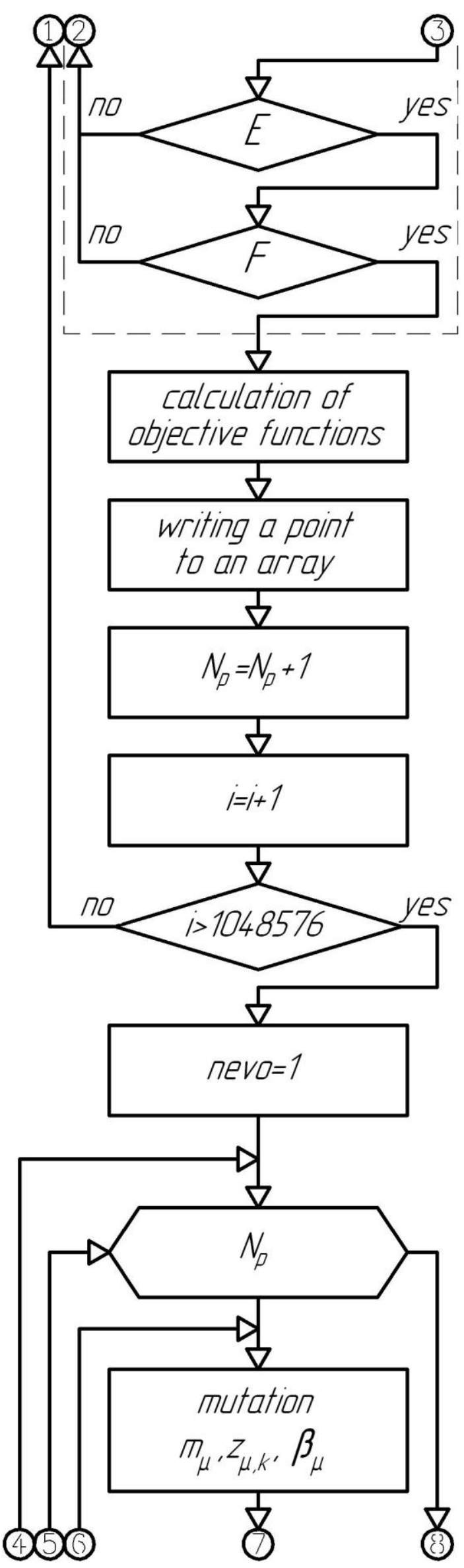

Fig. 1. EA (continuation). 


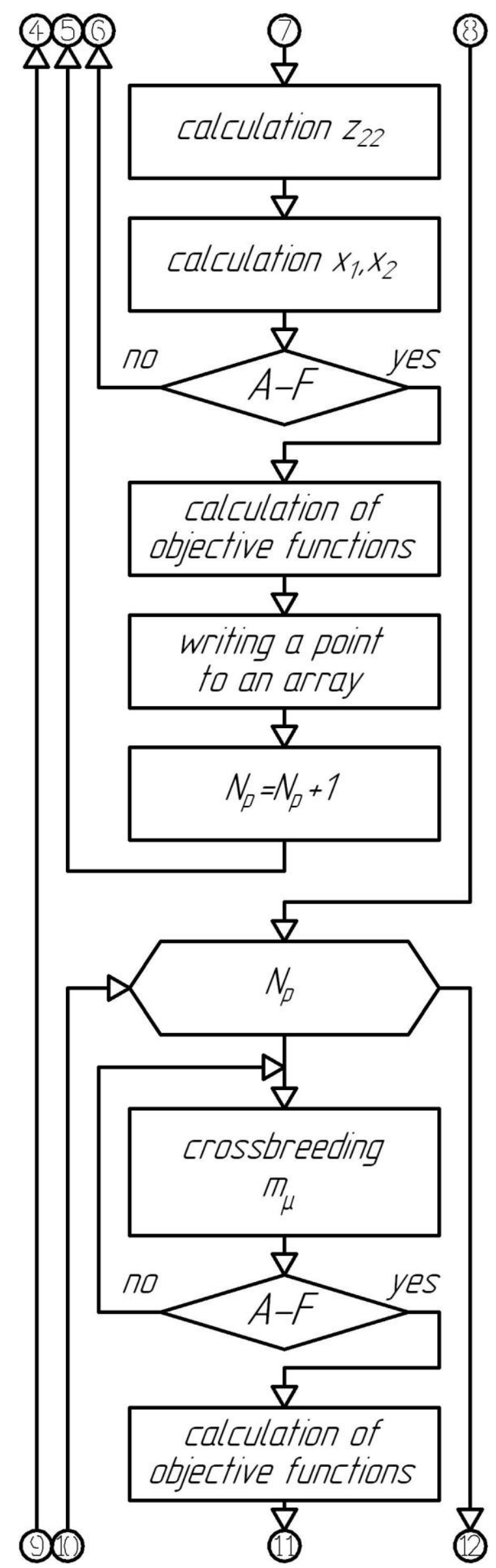

Fig. 1. EA (continuation).

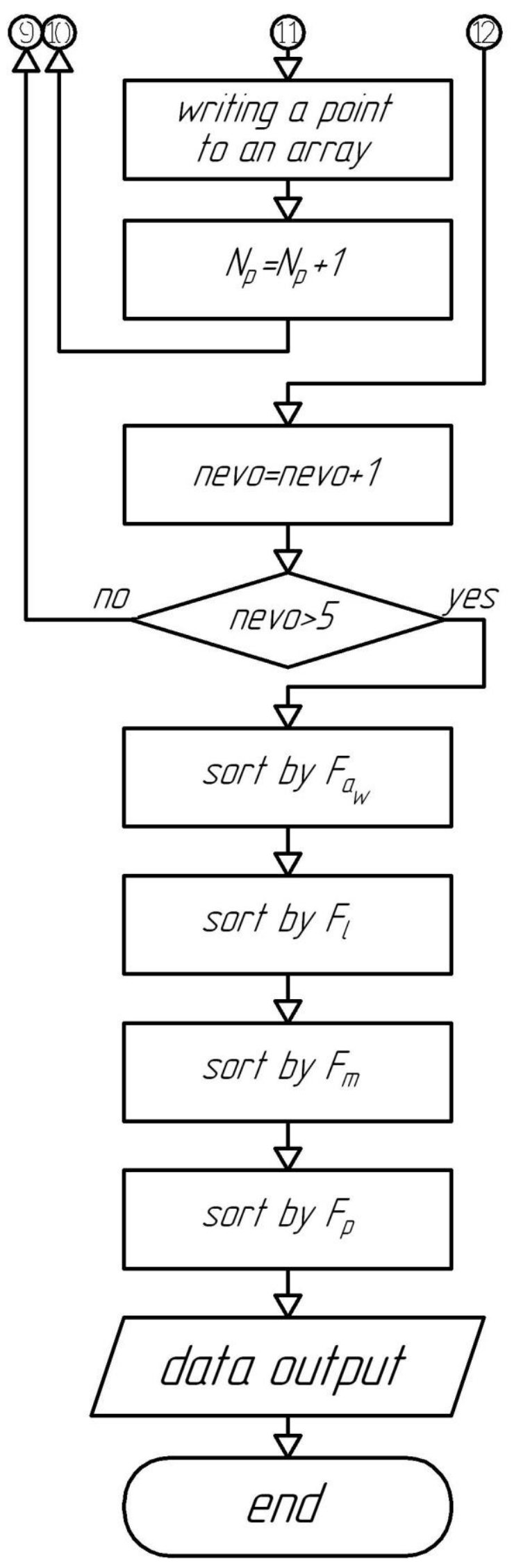

Fig. 1. EA (continuation). 


\section{Software implementations of the optimization algorithm}

In view of the presented algorithm, the optimal design of the reducers, which combines the given approach, the mathematical model of the problem (objective functions and limitations of the design parameters) and rational logical sequences of operations, developed an integrated program complex. Its implementation was carried out in the software Delphi 7, because the program language of this package enables to describe the algorithm qualitatively and rationally, it is quite easy to use.

The received program has a block-procedural structure, therefore it can be easily modified at the request of the designer, which enables to carry out optimally optimal design of other types of drives with fixed parallel shafts.

Also, one of the advantages of Delphi 7 is the ability to create a visual shell program as a standard window.
This allows the user to easily enter the necessary input data and easy view the data received.

\section{Examples of solving the problem of optimal design for two-stage reducers}

The first example of solving this problem is the cylindrical two-stage reducer to the flange motor, the KRZ, which has the following design parameters: $m_{1}=1.25 \mathrm{~mm}, m_{2}=1.5 \mathrm{~mm}, \beta_{1}=16^{\circ} 16^{\prime}, \beta_{2}=8^{\circ} 07^{\prime}, z_{11}=35$, $z_{12}=87, z_{21}=21, z_{22}=84, a_{w}=160 \mathrm{~mm}$.

Input data that correspond the design and technological requirements of the reducer: $T_{\text {inp }}=5 \mathrm{H} \cdot \mathrm{m}-$ torque on the input shaft; $n_{\text {inp }}=1500 \mathrm{rpm}$ - input shaft rotation frequency; $i_{\text {red }}=9.9428$ - the absolute value of gear ratio; resource $-10000 \mathrm{~h}$.

The results of the numerical experiment with limitation of equality for larger gear wheels in the meshes (A) and without this limitation are summarized in table 1 and table 2 respectively.

Table 1. Value of objective functions and design parameters (with limitation A)

\begin{tabular}{|l|c|c|c|c|c|c|c|c|c|c|c|c|c|c|c|c|c|c|c|}
\hline № & & $\min$ & $\max$ & $a_{w}$ & $F_{L}$ & $F_{M}$ & $F_{P}$ & $z_{11}$ & $z_{12}$ & $z_{21}$ & $z_{22}$ & $m_{1}$ & $m_{2}$ & $\beta_{1}$ & $\beta_{2}$ & $x_{1}$ & $x_{2}$ & $b_{w 1}$ & $b_{w 2}$ \\
\hline 1 & $a_{w}$ & 105.43 & 174.03 & - & 66.6 & 298.66 & 0.79 & 16 & 54 & 23 & 68 & 1.25 & 1.25 & 19 & 16 & 0.44 & 0.32 & 24.2 & 42.39 \\
\hline 2 & $F_{L}$ & 24.79 & 77.25 & 160.87 & - & 256.2 & 0.791 & 25 & 88 & 36 & 102 & 1.25 & 1.25 & 7 & 16 & 0.51 & 0.31 & 10.41 & 14.37 \\
\hline 3 & $F_{M}$ & 239.99 & 497.43 & 141.71 & 32.68 & - & 0.791 & 21 & 63 & 28 & 93 & 1.5 & 1.25 & 14 & 10 & 0.51 & 0.35 & 10.05 & 22.63 \\
\hline 4 & $F_{P}$ & 0.757 & 0.7926 & 137.17 & 41.84 & 353.08 & - & 20 & 81 & 14 & 34 & 1.25 & 3 & 13 & 6 & 0.51 & 0.2 & 15.1 & 26.74 \\
\hline
\end{tabular}

Table 2. Value of objective functions and design parameters (without limitation A)

\begin{tabular}{|l|c|c|c|c|c|c|c|c|c|c|c|c|c|c|c|c|c|c|c|}
\hline № & & $\min$ & $\max$ & $a_{w}$ & $F_{L}$ & $F_{M}$ & $F_{P}$ & $z_{11}$ & $z_{12}$ & $z_{21}$ & $z_{22}$ & $m_{1}$ & $m_{2}$ & $\beta_{1}$ & $\beta_{2}$ & $x_{1}$ & $x_{2}$ & $b_{w 1}$ & $b_{w 2}$ \\
\hline 1 & $a_{w}$ & 100.34 & 187.02 & - & 70.53 & 273.21 & 0.765 & 16 & 49 & 22 & 71 & 1.25 & 1.25 & 9 & 11 & 0.44 & 0.34 & 28.7 & 41.83 \\
\hline 2 & $F_{L}$ & 22.65 & 79.521 & 172.63 & - & 171.02 & 0.791 & 16 & 27 & 16 & 94 & 2 & 2.25 & 6 & 17 & 0.44 & 0.44 & 10.96 & 11.69 \\
\hline 3 & $F_{M}$ & 167.9 & 615.45 & 150.94 & 27.07 & - & 0.791 & 14 & 24 & 20 & 116 & 2.25 & 1.5 & 12 & 18 & 0.38 & 0.5 & 11.15 & 15.92 \\
\hline 4 & $F_{P}$ & 0.7557 & 0.7934 & 140.23 & 50.3 & 451.34 & - & 20 & 99 & 14 & 28 & 1.25 & 3 & 15 & 5 & 0.51 & 0.17 & 14.21 & 36.09 \\
\hline
\end{tabular}

According to the authors it is interesting to observe the dynamics of change in the number of offspring in the evolutionary process in steps, that is, the number of possible options for solving the problem. This dynamic can be conveniently presented in the form of a diagram, respectively for the variants with and without the limitation A, Fig. 2 and 3.

The authors proposed to present calculations results in graphical form for a more visual representation.

The calculations results are presented in the form of closed polygonal graph [10]. The graph is based on the axes, the number of which corresponds to the number of criteria.

Axes are locating in the plane of the sheet radially and uniformly. On each axis marked equal segments.

The beginning and end of the segments correspond to the limits of the existence of the criteria solution of within the task. The exception is the $F_{p}$ axis, which is displayed inversely.

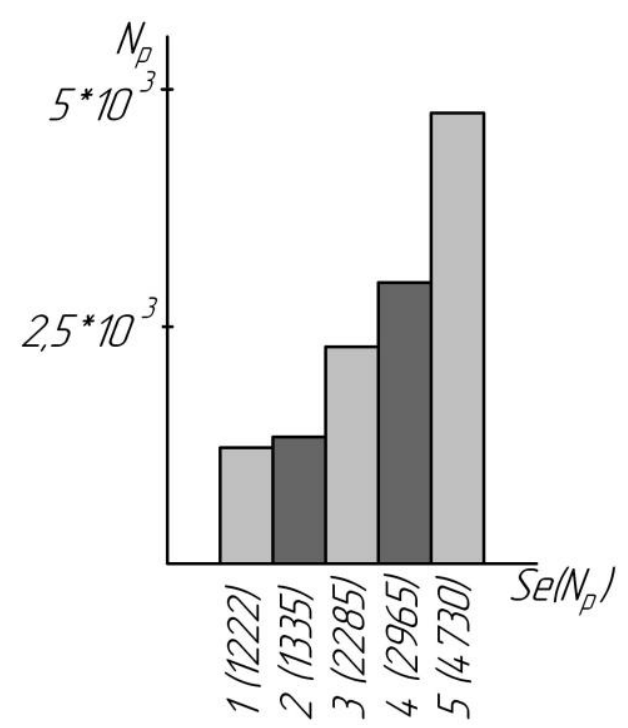

Fig. 2. Dynamics of change in the number of test points. 


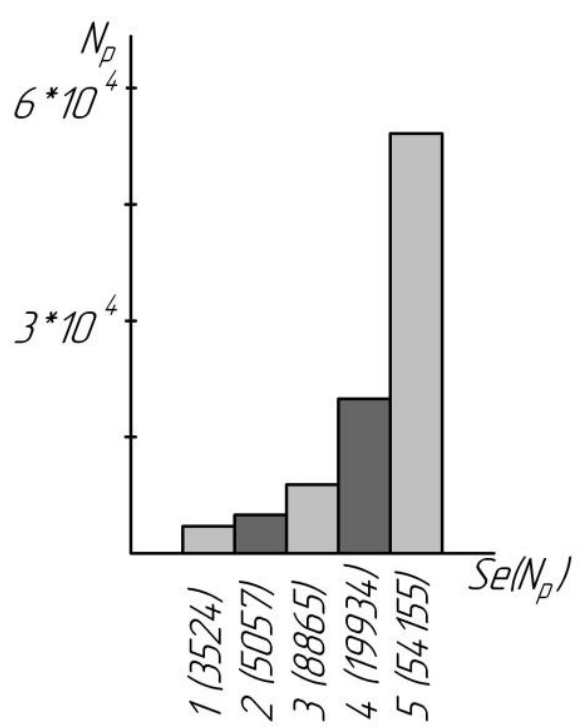

Fig. 3. Dynamics of change in the number of test points.

Closed polygonal graph criteria for the variant with and without the limitation $\mathrm{A}$ can be seen in the corresponding Fig. 4 and 5.

A second example of solving this problem is the cylindrical two-stage reducer ЦД2-85, which has the following design parameters: $m_{1}=3.5 \mathrm{~mm}, m_{2}=5 \mathrm{~mm}$, $\beta_{1}=8^{\circ} 06^{\prime}, \beta_{2}=8^{\circ} 06^{\prime}, z_{11}=36, z_{12}=162, z_{21}=28, z_{22}=170$, $a_{w}=850 \mathrm{~mm}$.

Input data that correspond the design and technological requirements of the reducer: $T_{\text {inp }}=552 \mathrm{H} \cdot \mathrm{m}$ - torque on the input shaft; $n_{\text {inp }}=1500 \mathrm{rpm}$ - input shaft rotation frequency; $i_{\text {red }}=27.31$ - the absolute value of gear ratio; resource $-10000 \mathrm{~h}$.

The results of the numerical experiment with and without limitation A are summarized in table 3 and table 4 respectively.

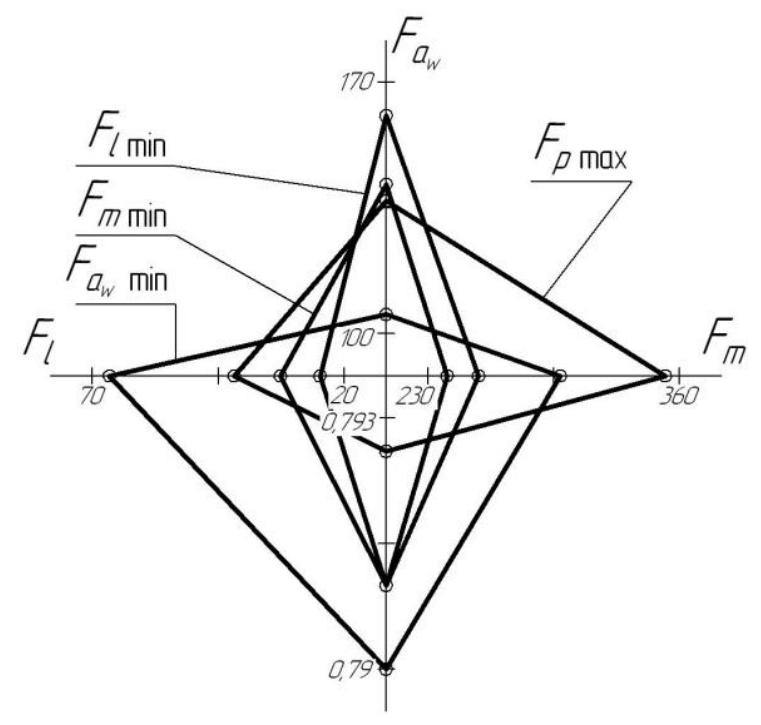

Fig. 4. Polygonal graphs solution.

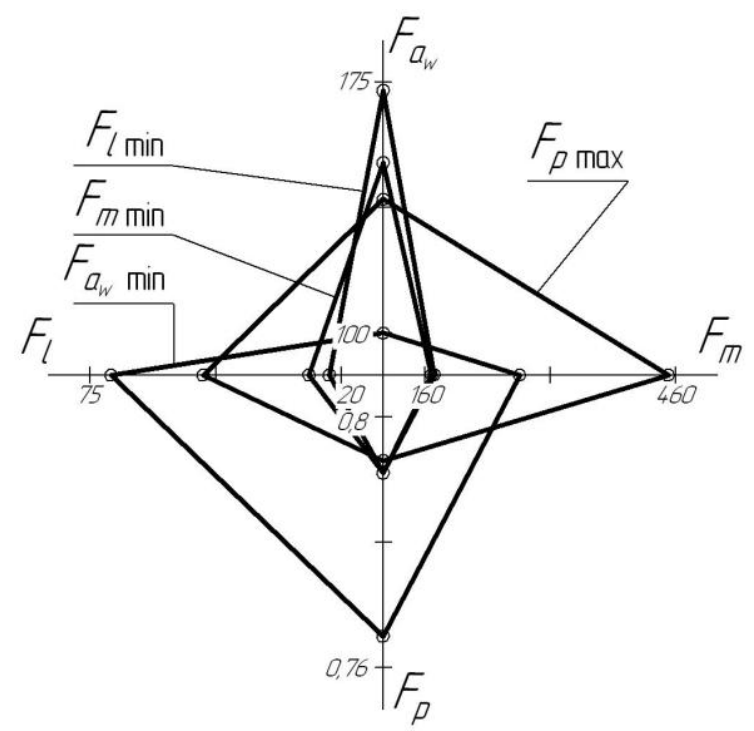

Fig. 5. Polygonal graphs solution.

Table 3. Value of objective functions and design parameters (with limitation A)

\begin{tabular}{|l|c|c|c|c|c|c|c|c|c|c|c|c|c|c|c|c|c|c|c|}
\hline № & & $\min$ & $\max$ & $a_{w}$ & $F_{L}$ & $F_{M}$ & $F_{P}$ & $z_{11}$ & $z_{12}$ & $z_{21}$ & $z_{22}$ & $m_{1}$ & $m_{2}$ & $\beta_{1}$ & $\beta_{2}$ & $x_{1}$ & $x_{2}$ & $b_{w 1}$ & $b_{w 2}$ \\
\hline 1 & $a_{w}$ & 725.12 & 1253.04 & - & 352.61 & 39724.82 & 0.732 & 21 & 136 & 31 & 131 & 4 & 5 & 8 & 7 & 0.51 & 0.41 & 122.98 & 229.62 \\
\hline 2 & $F_{L}$ & 118.07 & 376.18 & 1166.32 & - & 33181.81 & 0.791 & 23 & 145 & 40 & 173 & 6 & 6 & 7 & 14 & 0.51 & 0.41 & 43.17 & 74.89 \\
\hline 3 & $F_{M}$ & 29754.9 & 57003.38 & 853.34 & 253.3 & - & 0.759 & 26 & 125 & 34 & 193 & 5 & 4 & 13 & 13 & 0.51 & 0.49 & 46.08 & 207.22 \\
\hline 4 & $F_{P}$ & 0.7321 & 0.7948 & 877.34 & 262.54 & 42779.47 & - & 18 & 127 & 30 & 116 & 6 & 6 & 4 & 7 & 0.51 & 0.38 & 78 & 184.53 \\
\hline
\end{tabular}

Table 4. Value of objective functions and design parameters (without limitation A)

\begin{tabular}{|l|c|c|c|c|c|c|c|c|c|c|c|c|c|c|c|c|c|c|c|}
\hline № & & $\min$ & $\max$ & $a_{w}$ & $F_{L}$ & $F_{M}$ & $F_{P}$ & $z_{11}$ & $z_{12}$ & $z_{21}$ & $z_{22}$ & $m_{1}$ & $m_{2}$ & $\beta_{1}$ & $\beta_{2}$ & $x_{1}$ & $x_{2}$ & $b_{w 1}$ & $b_{w 2}$ \\
\hline 1 & $a_{w}$ & 704.85 & 1231.92 & - & 336.04 & 32418.84 & 0.724 & 27 & 143 & 35 & 180 & 3 & 4 & 13 & 14 & 0.51 & 0.46 & 122.48 & 213.55 \\
\hline 2 & $F_{L}$ & 113.99 & 376.18 & 1159.58 & - & 29848.57 & 0.791 & 28 & 158 & 38 & 184 & 5 & 6 & 5 & 16 & 0.51 & 0.45 & 43.19 & 71.8 \\
\hline 3 & $F_{M}$ & 21373.1 & 51369.26 & 979.89 & 159.21 & - & 0.792 & 42 & 144 & 25 & 199 & 3 & 6 & 11 & 15 & 0.51 & 0.51 & 45.86 & 113.35 \\
\hline 4 & $F_{P}$ & 0.7238 & 0.7937 & 857.21 & 252.17 & 37472.12 & - & 21 & 129 & 29 & 129 & 5 & 6 & 4 & 10 & 0.51 & 0.42 & 87.14 & 165.02 \\
\hline
\end{tabular}

Dynamics of change in the number of offspring in the evolutionary process in steps, presented in the form of a diagram, respectively for the variants with and without the limitation A, Fig. 6 and 7. 


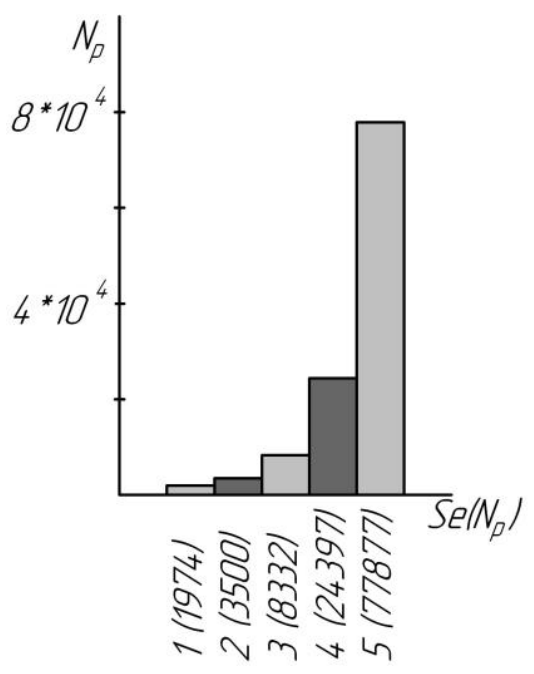

Fig. 6. Dynamics of change in the number of test points.

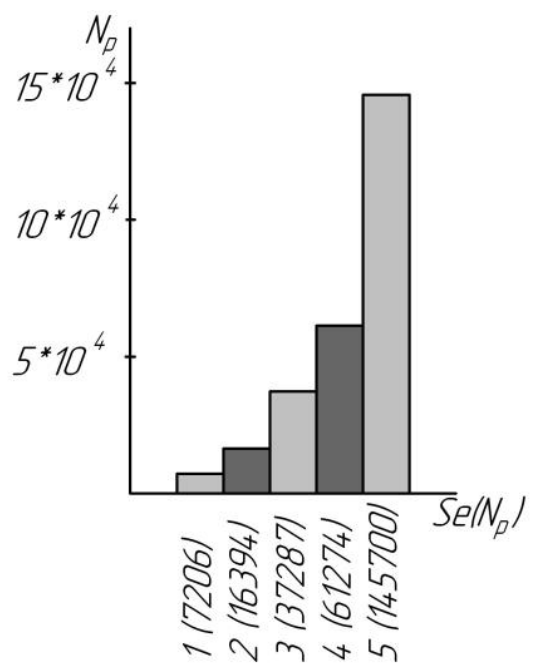

Fig. 7. Dynamics of change in the number of test points.

Closed polygonal graph criteria for the variant with and without the limitation $\mathrm{A}$ can be seen in the corresponding Fig. 8 and 9.

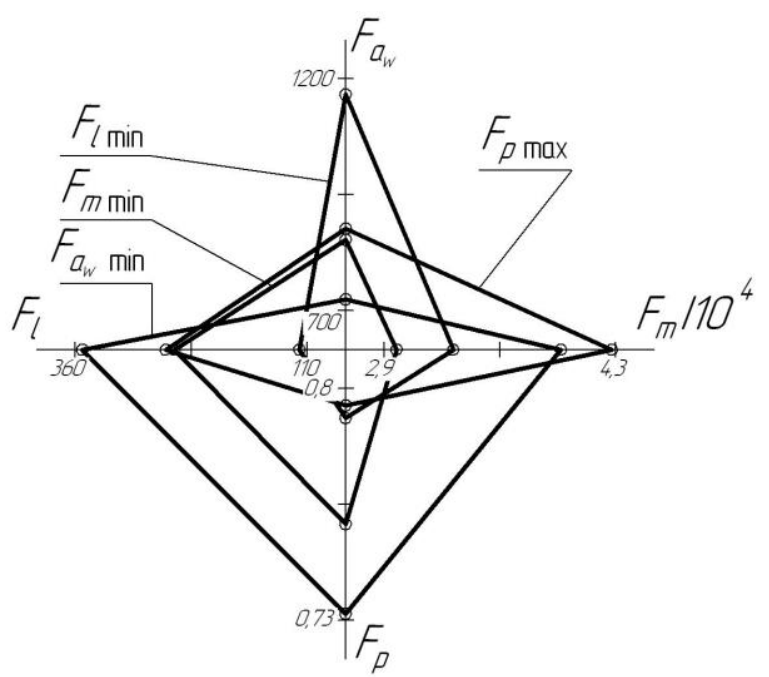

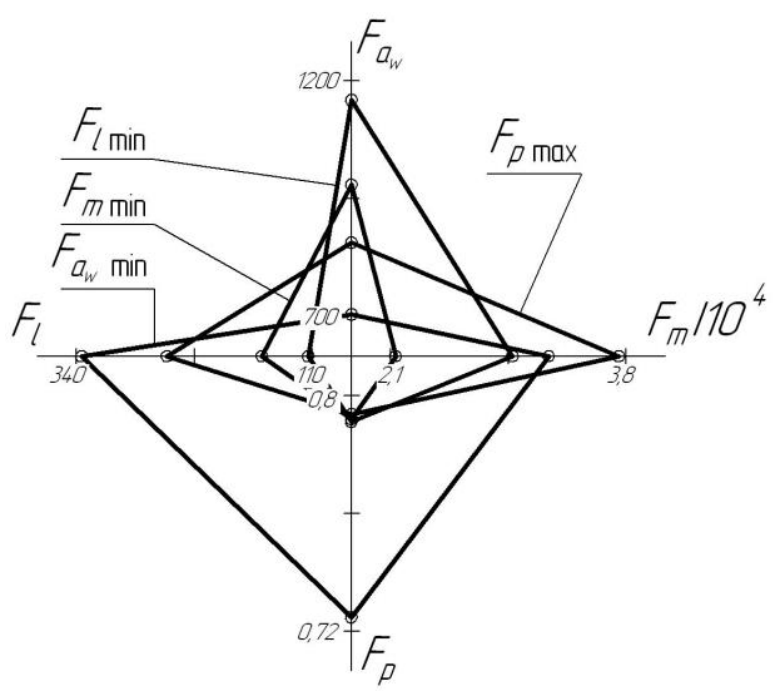

Fig. 9. Polygonal graphs solution.

\section{Conclusions:}

1. The relevance of the task is considered. The necessity to develop an approach to the design of drives with optimal design parameters with several criteria.

2.The given objective functions allow the designer to choose one or more quality criteria; the structure of the objective functions is logical and concise, and they can be supplemented by the necessary clarifying applications.

Checking limitations on design parameters (test points) is performed in a certain sequence, which allows to reduce the total optimization time due to timely screening of test points that do not meet number of requirements. The test point is sequentially checked for numerical and functional limitations on equality and inequality, and if it does not satisfy a certain requirement at some stage and the next check is started.

3. A modified evolutionary algorithm is proposed that allows to significantly increase the number of viable individuals, i.e. test points that satisfy the numerical and functional limitations of the problem, thereby eliminating the lack of limitation to the maximum possible number of trial points in the LP $\tau$ search. It is based on the screening of substandard points and the mandatory use of the mutation operator.

4. A numerical experiments were conducted. Its target is testing the proposed method for unifying the criteria and obtaining adequate output data. The universality and convenience of the proposed software system made it possible to quickly change the concept of a numerical experiment and to comfort vary input parameters.

5. Graphic options are provided for interpreting the output data to visualize the qualitative metrics of the solving process and directly the value and correlation of the quality criteria.

Fig. 8. Polygonal graphs solution. 


\section{References}

1. V. Platonov, H. Leyashvyly. Tracked and wheeled transport and traction machines (Moscow, Mashynostroenie Publ., 1986), (in Russian)

2. H. Rekleytys, A. Reyvyndran, K. Rjehsdel. Engineering Optimization (New York, A WileyInterscience Publ., 1983).

3. O. Bondarenko, O. Ustynenko. Optimization of coaxial step machine drives the weight and size characteristics on the example of three-shaft gearboxes. Bulletin of the NTU KhPI. Series: Engineering and CAD. Kharkiv, NTU "KhPI" Publ., 22 (2012). (in Ukrainian)

4. O. Bondarenko. Concentration of methods of LPTsearch and narrowing of nodes during optimization of gearboxes. Mechanics and machine building. Kharkiv, NTU "KhPI" Publ., 1 (2010). (in Ukrainian)

5. O. Bondarenko, O. Ustynenko, V. Serykov. The rational design of two-stage cylindrical gear reducers taking into account level of gears tension. Bulletin of the NTU KhPI. Coll. of scientific papers. Series: Problems of mechanical drive. Kharkiv, NTU "KhPI" Publ., 35 (2015). (in Ukrainian)
6. O. Ustynenko, O. Bondarenko, V. Serykov. Ability to use and adapt genetic algorithms for rational gear design cylindrical reducers and gearboxes. Bulletin of the NTU KhPI. Coll. of scientific papers. Series: Engineering and CAD. Kharkiv, NTU "KhPI" Publ., 7 (2019). (in Ukrainian)

7. Trần Minh Kiệt. Design of two-stages helical gearboxes in order to get minimal length of gearboxes. Journal of Marine Science and Technology (April 2015).

8. L. Tudose, O. Buiga, C. Stefanache, A. Sobester. Automated optimal design of a two-stage helical gear reducer. Structural and Multidisciplinary Optimization manuscript (authors' post-print) (Sept. 2010).

9. O. Ustynenko, O. Bondarenko. The use of pseudorandom sequences in evolutionary algorithms in rational design of gear cylindrical reducers and gearboxes. Bulletin of the NTU KhPI. Coll. of scientific papers. Series: Engineering and CAD. Kharkiv, NTU "KhPI" Publ., 1 (2019). (in Ukrainian)

10. O. Ustinenko, O. Bondarenko, I. Klochkov, V. Serykov. Multi-criteria optimization of tracked vehicle transmissions. Proc. of 6th International BAPT Conference Power Transmissions, 1922.06.2019, Varna, 1 (2019). 